Article

\title{
Application of Infrared Spectroscopy in Prediction of Asphalt Aging Time History and Fatigue Life
}

\author{
Yuan Yan, Yanmei Yang, Maoping Ran *, Xinglin Zhou, Lanlin Zou and Minrui Guo
}

School of Automotive and Traffic Engineering, Wuhan University of Science and Technology, Wuhan 430065, China; sahara1990@163.com (Y.Y.); yym6255@163.com (Y.Y.); zhouxinglin@wust.edu.cn (X.Z.); zoulanlin@wust.edu.cn (L.Z.); 20131293@huanghuai.edu.cn (M.G.)

* Correspondence: ranmaoping@wust.edu.cn

Received: 5 August 2020; Accepted: 28 September 2020; Published: 5 October 2020

\begin{abstract}
Based on attenuated total reflection Fourier-transform infrared (ATR-FTIR) spectroscopy and principal component analysis (PCA), four kinds of asphalt (two kinds of matrix asphalts, TPC70\# and JL70\# and two kinds of modified asphalts, SBS-1 and SBS-2) were aged using the rolling thin-film oven test (RTFOT). The multiple stress repeated creep recovery test (MSCR) was carried out on an AR1500ex dynamic shear rheometer (DSR). The PCA was carried out on the attenuated total reflection infrared spectrum of a pretreated aged asphalt, the principal component factors (oxidation factor and component change factor) were determined, the comprehensive index $F$ was calculated, and a prediction model of aging time history was established and verified. A prediction model for the aging asphalt recovery rate $R$ was established based on the functional group index. The relationship between the recovery rate $R$ and aging time history of each aging asphalt was analyzed and the fatigue life of each aging asphalt based on the rate of dissipated energy change (RDEC) was compared. The correlation between the comprehensive index $F$ and fatigue life of asphalt was also analyzed. The results show that the order of resistance to the high-temperature deformation of each aging asphalt is as follows: SBS-2 > SBS-1 > TPC70\# > JL70\#; the prediction model of aging asphalt recovery rate $R$ based on functional group index has good reliability. Taking the loading cycle corresponding to the sudden increase of the inflection point of the curve, i.e., the fatigue life $\mathrm{N}_{\mathrm{RDEC}}$, as the evaluation index, the fatigue life order of each aging asphalt is SBS-1 > SBS-2 > JL70\# > TPC70\#. There is a positive correlation between the fatigue life of aged asphalt and the comprehensive index $\mathrm{F}$ of asphalt. The correlation degree $R^{2}$ is 0.85 ; i.e., with the increase of the comprehensive index $\mathrm{F}$ of asphalt, its fatigue life also increases. It was found that it is feasible to analyze the aging time history and fatigue life of asphalt by infrared spectroscopy, and it can provide a rapid and non-destructive prediction method for the practical engineering application of asphalt.
\end{abstract}

Keywords: infrared spectrum; aging time history; fatigue life; asphalt recovery rate; principal component analysis

\section{Introduction}

Asphalt aging is an important factor that shortens the life of asphalt pavements [1]. Consequently, research on asphalt aging time and fatigue life is of great significance to improve the durability of asphalt pavements; however, asphalt performance is the most difficult factor to monitor in the construction asphalt paving process, and its technical indicators need to be tested according to specifications [2]. The traditional test not only takes a long time and damages the detected road sections to varying degrees, but the measured results can vary when sampling positions are slightly different. Therefore it is impossible to accurately and comprehensively obtain the dynamic usage of road sections. The testing process of infrared spectroscopy technology is convenient, fast, pollution-free, and does not damage 
the road surface. There is no need to pretreat the detected samples, and many performance indexes can be calculated and studied through the detected spectral data; therefore, the infrared spectroscopy detection method not only saves time, but also reduces the cost of research [3]. Some scholars [4-9] selected characteristic peaks such as carbonyl and sulfoxide groups to study the characterization of asphalt aging behavior; these authors aim to use infrared spectroscopy to study aging asphalt, the effect of modifier on asphalt, and the optimal amount of modifier; however, they only considered the characteristic absorption peaks that have undergone significant changes, selected characteristic peak height (ratio) or peak area (ratio), and characterization parameters to establish a simple linear regression relationship, which may result in an insufficient infrared spectrum analysis of asphalt aging time and rheological performance.

With the development of chemometrics, the combination of spectral technology and chemometrics has been widely used in various fields. Some scholars have applied this method to study the anti-aging performance of asphalt. Researchers [10-13] have used principal component analysis (PCA), linear discriminant analysis (LDA), partial least squares (PLS), and other methods to analyze the infrared spectrum data of asphalt. They found that the stoichiometric analysis of the infrared spectrum can distinguish asphalt samples through refineries; PLS and LDA of infrared spectral data can predict the penetration logarithm, softening point, mass content, and rheological parameters of asphalt; however, the above tests have higher requirements with regard to the source and type of asphalt and have certain limitations on the parameters of different types of asphaltene content, softening point, the logarithm of penetration, composite shear modulus, and phase angle at different temperatures.

An increasing number of studies have shown that the three traditional indicators of asphalt (penetration, ductility, and softening point) cannot accurately characterize its anti-aging properties. Currently, the widely used evaluation indices of asphalt aging include quality change, residual penetration ratio, residual ductility ratio, residual viscosity ratio, viscosity increase, softening point increase, etc. Some authors [14-16] have carried out relevant research on these indices and used each aging index to analyze the aging trend of asphalt to evaluate the anti-aging performance of asphalt. In addition, some researchers $[17,18]$ believe that the colloidal instability index $I_{\mathrm{c}}$ can reflect the peptization ability of soft asphalt well. The larger its value, the more gel-like asphalt tends to be, and its colloidal structure becomes more unstable, thus, the peptization ability and anti-aging ability deteriorate.

In this study, based on attenuated total reflection Fourier-transform infrared (ATR-FTIR) spectroscopy and PCA, the infrared spectral data of four kinds of aged asphalt were qualitatively and quantitatively analyzed, the comprehensive index $F$ was calculated, a prediction model of asphalt aging time history was established and verified, and a prediction model of asphalt recovery rate $R$ was also established using a functional index. In this study, the relationship between the recovery rate $R$ and the aging time history of each aging asphalt was also analyzed; then, the fatigue life of each was compared. Finally, to rapidly predict the aging time and fatigue life of asphalt, the correlation between asphalt fatigue life and aging time history based on the rate of dissipated energy change.

\section{Test Methods}

\subsection{Rolling Thin-Film Oven Test (RTFOT)}

In this test, two base asphalts of TPC70\# and JL70\# and SBS (I-D) modified asphalts of two different base asphalts were selected, which were denoted as SBS-1 and SBS-2, respectively. They are all commercial asphalts, among which TPC70\# asphalt comes from Jiangsu Zhenjiang Topco Asphalt Co., Ltd., and JL70\# asphalt comes from Nanjing Jinling Asphalt Industrial Co., Ltd. According to the current "Testing Regulations for Asphalt and Asphalt Mixtures of Highway Engineering" (JTG E20-2011) [19], the technical indicators of the above four types of asphalts were tested, as shown in Table S1 (see supplementary file Tables.pdf) - T0604 is the asphalt penetration test in JTG E20-2011, T0605 is the asphalt ductility test, T0606 is the asphalt softening point test, T0625 is the asphalt Brinell 
rotational viscosity test, and T0610 is the asphalt rotary film heating test. Their technical indicators complied with the specifications.

Five different aging time histories were selected to carry out the rolling thin-film oven test (RTFOT, Nanjing Xiaoxiao Instrument Equipment Co., Ltd., Nanjing, China) on the above four asphalt samples. Next, $35 \mathrm{~g}$ asphalt samples were placed into an open glass bottle with a diameter of $64 \mathrm{~mm}$ and a height of $140 \mathrm{~mm}$, then quickly inserted into a rolling thin-film oven, with the temperature set at $163{ }^{\circ} \mathrm{C}$ and the rotating speed at $15 \mathrm{r} / \mathrm{min}$. At the same time, $4000 \mathrm{~mL} / \mathrm{min}$ of hot air was continuously blown in. After reaching the required test time, the sample bottle was removed and the bitumen in the bottle was poured into the container. All the sample bottles should be removed within $5 \mathrm{~min}$, and all the aged asphalt should be tested within $72 \mathrm{~h}$. The test device is shown in Figure 1.

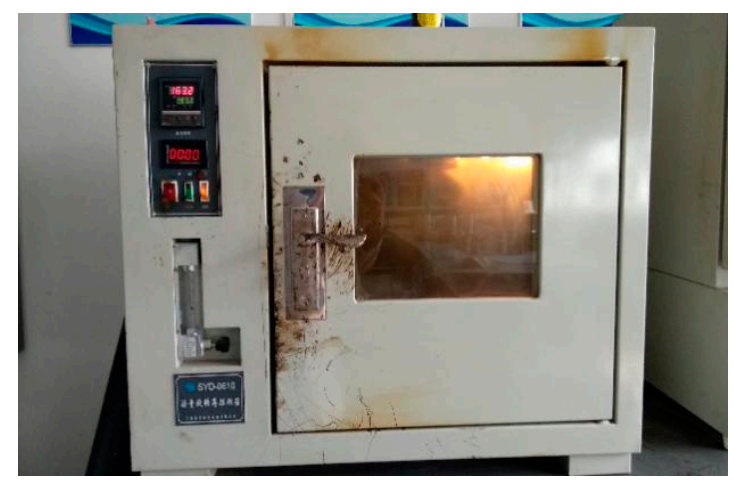

Figure 1. Rolling thin-film oven test (RTFOT).

The aging time of five RTFOT tests are as follows: A. the aging time was $0 \mathrm{~min}$; B. the aging time was $85 \mathrm{~min}$; C. the aging time was $120 \mathrm{~min}$; D. the aging time was $240 \mathrm{~min}$; E. the aging time was $360 \mathrm{~min}$. A total of 20 asphalt samples were produced, as shown in Table S2 (see supplementary file Tables.pdf).

\subsection{Infrared Spectrum Acquisition}

We used the Thermo Scientific Nicolet iS50 Fourier transform infrared spectrometer (Shanghai Simiao Analytical Instrument Co., Ltd., Shanghai, China), and selected the ATR accessory to collect infrared spectrograms of aged asphalt samples, as shown in Figure 2. The Fourier infrared spectrometer needs to be preheated for at least $30 \mathrm{~min}$ in advance. Before each measurement, a background scan was performed. The acquisition parameters were set to a resolution of $4 \mathrm{~cm}^{-1}$, the number of scans was 32, and the test range was between 500 and $4000 \mathrm{~cm}^{-1}$. The aging asphalt in the flowing state at the end of the aging simulation experiment was directly sampled to avoid reheating. The asphalt samples were smear-melted onto a dry and clean $\mathrm{SiO}_{2}$ glass sheet of $20 \times 20 \times 1 \mathrm{~mm}^{3}$ so that the glass sheet was completely covered and the asphalt surface on the glass sheet was as smooth as possible. Each sample was duplicated 3 times and loaded 3 times during collection. For each sample, 9 spectra were recorded, which resulted in a collection of 180 spectrograms $(20 \times 9)$ in total, thus ensuring the repeatability of the asphalt samples and a reduction in the instability of the measurement environment. 


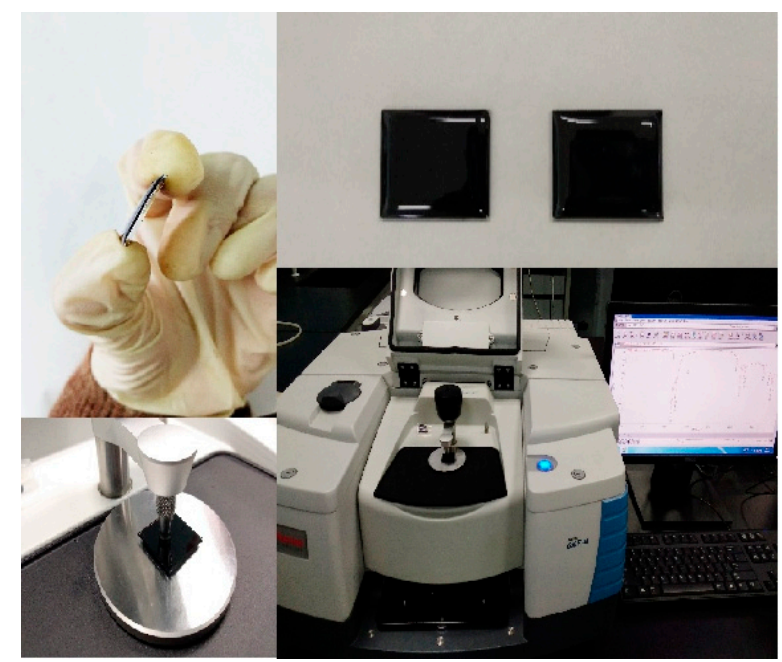

Figure 2. Infrared spectrum acquisition.

\subsection{Multiple Stress Creep Recovery (MSCR) Test}

To evaluate the ability of the aging asphalt to resist permanent deformation, the aging residue of the asphalt rotary oven was subjected to a multi-stress creep recovery (MSCR) test. The AR1500ex (Beijing Ouya Xingyu Technology Co., Ltd., Beijing, China) dynamic shear rheometer (DSR) was used for MSCR. For the ASTMD7405 [20], a $25 \mathrm{~mm}$ rotor was selected, and the gap between the rotor and the lower parallel plate of the DSR fixture was set to $1 \mathrm{~mm}$. The multi-stress creep recovery test was carried out at five temperatures: $64,70,76,82$, and $88^{\circ} \mathrm{C}$. The dynamic shear rheometer applied a creep stress of 0.1 and $3.2 \mathrm{kPa}$ to asphalt samples successively, and each stress level was loaded and unloaded in a time ratio of $1 \mathrm{~s}: 9 \mathrm{~s}$ for 10 cycles [21]. Repeated tests of the three asphalt samples were carried out at different temperatures to ensure the repeatability of the tests and the final test result was the average of the three repeated test results.

The final evaluation indices of the MSCR test were unrecovered creep compliance $J_{\text {nr }}$ and recovery rate $R$. The smaller the $J_{\mathrm{nr}}$ value and the larger the $R$-value, the better the high-temperature performance of asphalt, i.e., the better the deformation resistance and recovery ability. The calculation formulas of the two are shown below.

$$
\begin{gathered}
J_{\mathrm{nr}}(\sigma, N)=\frac{\varepsilon_{10}}{\sigma} \\
R(\sigma, N)=\frac{\varepsilon_{1}-\varepsilon_{10}}{\varepsilon_{1}} \times 100 \% \\
J_{\mathrm{nr}}=\sum_{N=1}^{10} J_{\mathrm{nr}}(\sigma, N) / 10 \\
R=\sum_{N=1}^{10} R(\sigma, N) / 10
\end{gathered}
$$

where $\sigma$ is the applied stress level, which is 0.1 and $3.2 \mathrm{kPa} ; \mathrm{N}$ is the loading period; $J_{\mathrm{nr}}(\sigma, N)$ is the unrecovered creep compliance of the nth cycle. $R(\sigma, N)$ is the recovery rate of the nth cycle; $\varepsilon_{10}$ is the unrecovered strain at the end of the recovery phase of the nth cycle (i.e., the $10 \mathrm{~s}$ ) relative to that before loading; $\varepsilon_{1}$ is the strain at the end of loading for the nth cycle (i.e., the first $1 \mathrm{~s}$ ) relative to that before loading; $J_{\mathrm{nr}}$ is the average unrecovered creep compliance; $R$ is the average recovery rate. 


\section{Results and Analysis}

\subsection{Infrared Spectrum Analysis of Aged Asphalt}

\subsubsection{Qualitative Analysis}

To eliminate the presence of interference from noise and physical factors in spectral data and ensure spectral information reflects the truest spectral characteristics of samples to the greatest extent, the original spectral data should be preprocessed first. According to the interference in spectrum, $180(20 \times 9)$ spectrograms (as detailed in Section 2.2) were preprocessed by the SNV (smoothing of normalized vector) method (the number of smoothing points was 5) and baseline correction. Finally, the average absorption intensity of 9 preprocessed infrared spectrograms of each asphalt sample was taken as the final spectra data of the asphalt sample. The spectral data of 20 asphalt samples were imported into the Origin 8.0 (Originlab Company, Northampton, MA, USA) and the attenuated total reflection Fourier infrared spectrogram of all asphalt was drawn as shown in Figure 3.

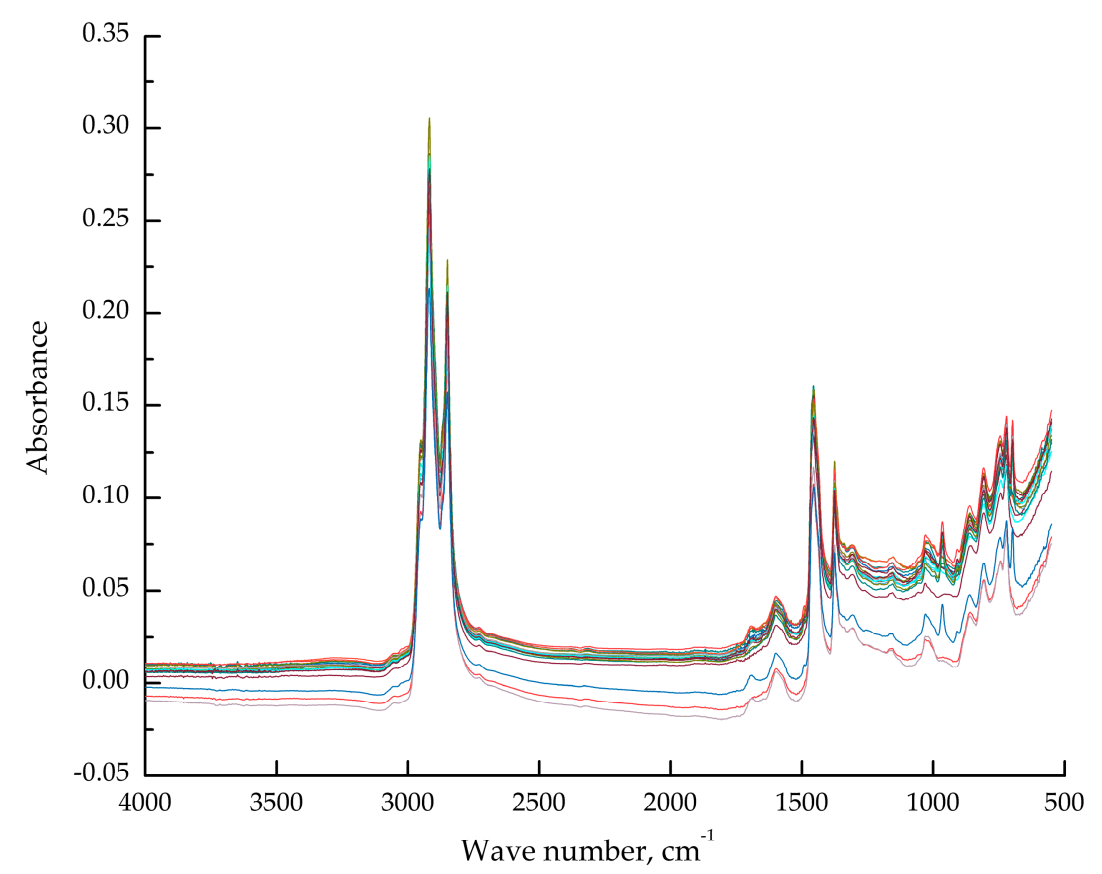

Figure 3. Attenuated total reflection Fourier infrared spectroscopy of 20 asphalt samples.

Figure 3 shows that the infrared spectra shapes of 20 samples of four kinds of asphalt are similar, and the characteristic absorption peaks and functional groups have similar wave numbers; however, due to the different properties of asphalt samples, the peak heights of some characteristic absorption peaks, i.e., absorbance, are quite different. The characteristic absorption peaks were chosen as research parameters to explore whether asphalt types can be distinguished, to some extent, according to characteristic absorption peaks.

To analyze the change of infrared spectrum characteristics of asphalt samples with different aging degrees, the infrared spectrum comparison diagrams of four types of asphalt with different aging degrees are drawn, as shown in Figure 4. 

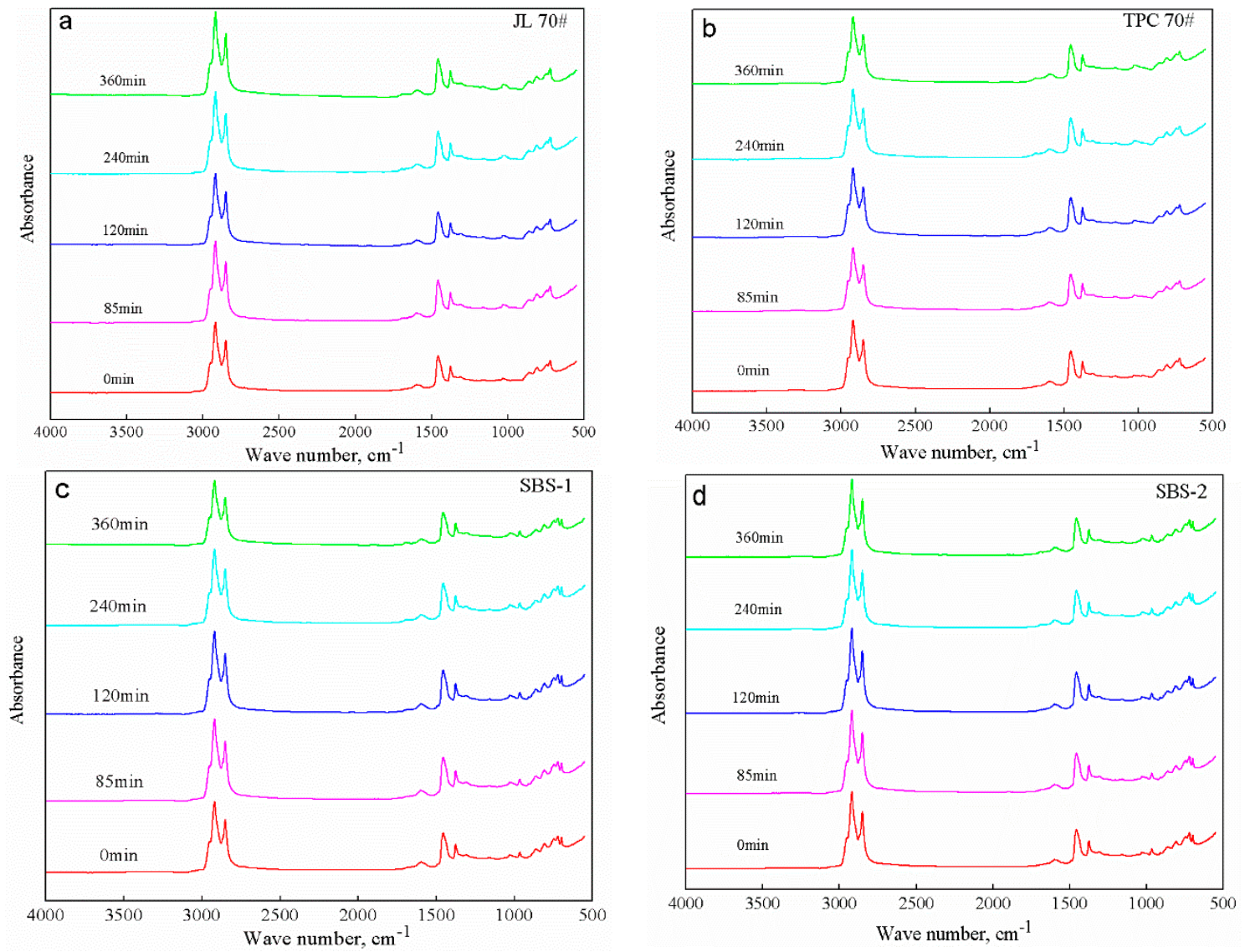

Figure 4. The infrared spectrum diagrams of four types of asphalt with different aging time. (a) JL70\#; (b) TPC70\#; (c) SBS-1; (d) SBS-2.

According to Figure 4, JL70\# and TPC70\# have the same peak positions and changes, and there are 13 evident characteristic absorption peaks. The SBS-1 and SBS-2 asphalt have the same peak position and change. Compared with the base asphalt, in addition to the 13 absorption peaks in total, there are two more obvious characteristic absorption peaks at 699 and $966 \mathrm{~cm}^{-1}$, respectively; therefore, the base asphalt and SBS-modified asphalt can be distinguished by the presence or absence of these two characteristic absorption peaks. The absorption peak of the carbonyl group $(\mathrm{C}=\mathrm{O})$ appeared at $1700 \mathrm{~cm}^{-1}$ due to the oxidation reaction of carbon and corresponding to the short-term aging, and the absorption peak became more significant with the increase in aging time. However, the rate of change of the absorption peak of several asphalts was different. The absorption peak at $1030 \mathrm{~cm}^{-1}$ corresponds to the sulfoxide group $(\mathrm{S}=\mathrm{O})$. The absorption peak of the four kinds of asphalt is weak, and intensity of the absorption peak increases with the aging time. However, the rates of change of the absorption peaks of several kinds of asphalt are different. Both the sulfoxide group and carbonyl group change significantly with the increase in aging time, thus, they can reflect the aging degree of asphalt to a certain extent.

\subsubsection{Quantitative Analysis}

Research $[22,23]$ shows that the change of the four-component content during the aging of asphalt can reflect the aging degree of asphalt. Some researchers [24] analyzed the RTFOT and pressure aging vessel (PAV) aging effects of the asphalt binders to understand the functional polar groups in the asphalt system under short- and long-term aging conditions, respectively. They found that the functional groups containing the carbonyl group in the asphalt binders can be also used to correlate to the oxidation extent of asphalt binders. According to references [25,26], the aliphatic functional groups $\left(\mathrm{CH}_{2}, \mathrm{CH}_{3}\right)$ at 2920 and $2820 \mathrm{~cm}^{-1}$ and asymmetric aliphatic functional groups $\left(\mathrm{C}^{-} \mathrm{CH}_{3}\right)$ at 1376 and $1456 \mathrm{~cm}^{-1}$ 
were used in this study, which reflected the change of asphalt saturation. The changes in the aromatic functional group at $1600 \mathrm{~cm}^{-1}$, carbonyl functional group at $1700 \mathrm{~cm}^{-1}(\mathrm{C}=\mathrm{O})$, and sulfoxide functional group at $1030 \mathrm{~cm}^{-1}(\mathrm{~S}=\mathrm{O})$, show the change of the aromatic ring components (aromatic components, gum, and asphaltene), which are the main objects of the quantitative analysis.

The infrared spectrograms of 20 asphalt samples were quantitatively analyzed according to Lambert-Beer's law [27]. First, the correction baseline is the tangent of the lowest point on both sides of the characteristic absorption peak, and the area enclosed by the baseline and the spectral curve is the peak area of the absorption peak [28]. The peak areas of 15 obvious characteristic absorption peaks of each asphalt were calculated by OMNIC 8.2 (Nicolet). To explore the best quantitative analysis method, the selected benchmark for the study was [4,5]: A1, the sum of peak areas in the range of $650 \sim 1400 \mathrm{~cm}^{-1}$; $\mathrm{A} 2$, the sum of peak areas in the range of $1400 \sim 4000 \mathrm{~cm}^{-1} ; \mathrm{A} 3$, full spectrum in the range of $650 \sim 4000 \mathrm{~cm}^{-1}$; A4, the sum of peak areas in the range of $2920 \sim 2850 \mathrm{~cm}^{-1}$. By comparing the different analysis methods, the peak area ratio of each characteristic absorption peak, i.e., functional group index, is defined as follows:

$$
\left\{\begin{array}{l}
I_{\mathrm{C}=\mathrm{O}}=A_{1700} / \sum A_{i} \\
I_{\mathrm{S}=\mathrm{O}}=A_{1030} / \sum A_{i} \\
I_{\mathrm{B}}=\left(A_{2920}+A_{2850}\right) / \sum A_{i} \\
I_{\mathrm{B}, \mathrm{a}}=\left(A_{1376}+A_{1456}\right) / \sum A_{i} \\
I_{\mathrm{Ar}}=A_{1600} / \sum A_{i}
\end{array}\right.
$$

where $I_{\mathrm{C}=\mathrm{O}}$ and $I_{\mathrm{S}=\mathrm{O}}$ are carbonyl and sulfoxide functional group indices, respectively; $I_{\mathrm{B}}$ and $I_{\mathrm{B}, \mathrm{a}}$ are aliphatic functional group and asymmetric aliphatic functional group indices; $I_{\mathrm{Ar}}$ is aromatic functional group index; $\mathrm{A}_{2920}, \mathrm{~A}_{2850}, \mathrm{~A}_{1700}, \mathrm{~A}_{1600}, \mathrm{~A}_{1456}, \mathrm{~A}_{1376}$, and $\mathrm{A}_{1030}$ are the corresponding peak areas at wavenumbers of $2920,2850,1700,1600,1456,1376$, and $1030 \mathrm{~cm}^{-1}$, respectively. $\sum A_{i}$ is the sum of peak areas under the ith benchmark $(i=1,2,3,4)$, and the specific values are shown in Table S3 (see supplementary file Tables.pdf).

From the above analysis, it can be seen that the peak area of functional groups is not related to the selection of reference standards, and selecting different reference standards will make the change rule of the functional group index different or even opposite with the increase in aging time. Asphalt reacts with oxygen to produce carbonyl and sulfoxide groups, which results in an increase in the functional index of carbonyl and sulfoxide groups as the asphalt ages [29]. Some scholars [30] calculated carbonyl and sulfoxide indices using different baseline correction methods and tangential and baseline integration, respectively. They found that the sulfoxide indices calculated by both methods were not affected by the baseline correction method used. According to Formula (2), the functional group indices of asphalt samples under different benchmarks were calculated, and the changing trend of carbonyl and sulfoxide functional group indices is shown in Figure 5.

Figure 5 shows that the carbonyl index of the four types of asphalt increases under the selected four reference standards, whereas the sulfoxide group of TPC 70\# asphalt shows a negative growth within the aging time of 120 240 min under the benchmarks A2, A3, and A4, which does not conform to the aging mechanism of asphalt; therefore, A1 $\left(650 \sim 1400 \mathrm{~cm}^{-1}\right)$ was selected as the reference standard in this work so that the changes of functional groups in the asphalt aging process can be accurately and reasonably characterized. The functional group indices of 20 asphalt samples under the A1 standard are shown in Table S4 (see supplementary file Tables.pdf). 

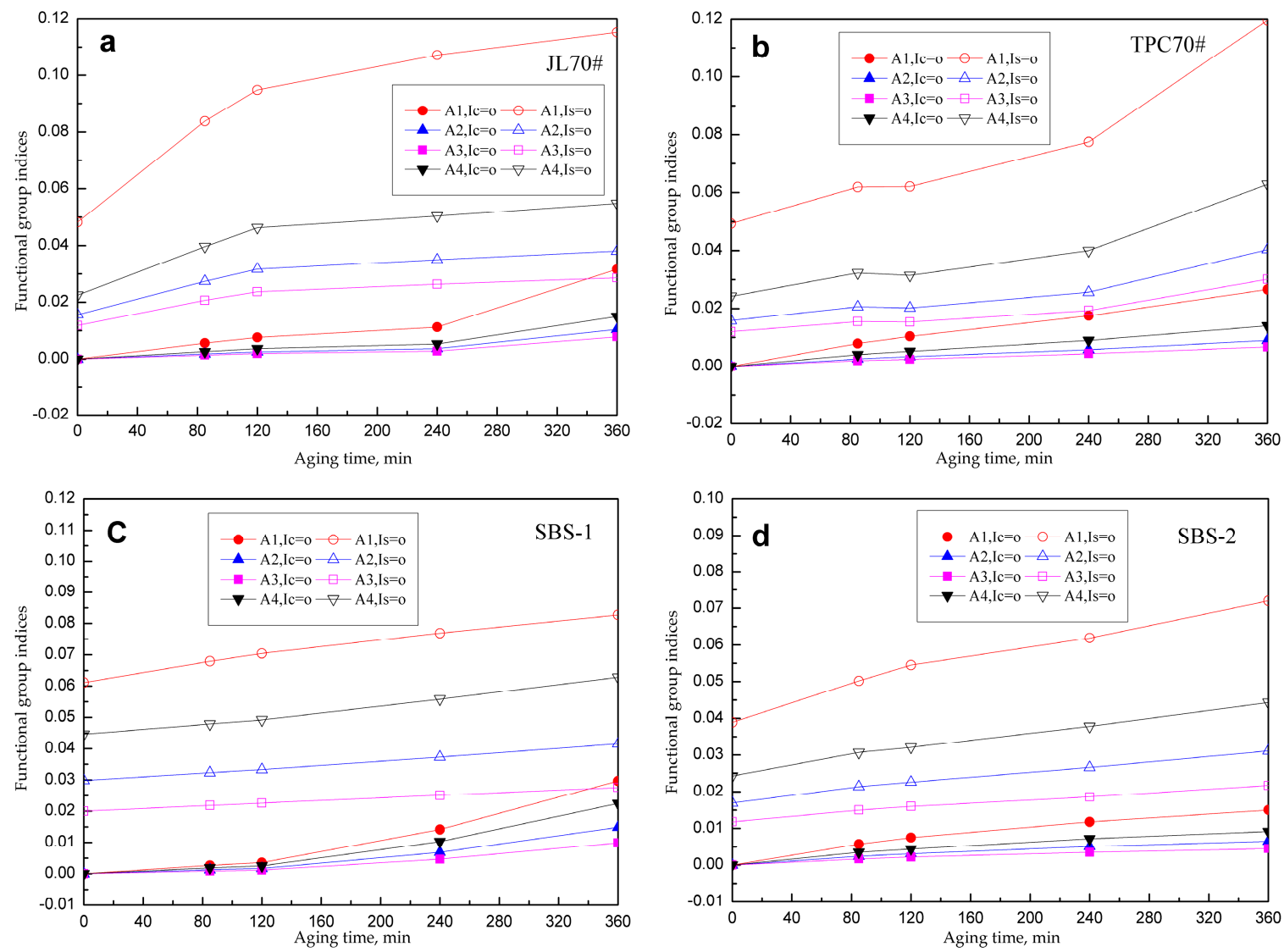

Figure 5. The changing trend of the carbonyl and sulfoxide functional group indices of all asphalt samples. (a) JL70\#; (b) TPC70\#; (c) SBS-1; (d) SBS-2.

\subsection{MSCR Test Results and Analysis of Aged Asphalt}

The unrecovered creep compliance $J_{\mathrm{nr}}$ and recovery rate $R$ of 20 aged asphalt samples under different temperatures and stress conditions were calculated according to Equations (1)-(4). By comparing and analyzing the influence of temperature and stress on asphalt in Figure 6, it is found that $J_{\mathrm{nr} 0.1}$ of JL70\# and TPC70\# are slightly smaller than $J_{\mathrm{nr} 3.2}$ and $R_{0.1}$ is slightly larger than $R_{3.2}$ under any temperature, which shows that the sensitivity of matrix asphalt to applied stress is weak. However, after $70^{\circ} \mathrm{C}, J_{\mathrm{nr}}$ and $R$ of the two modified asphalts differ greatly under different stress levels and change with temperature, which indicates that the modified asphalt is sensitive to temperature and stress. As a result, $70{ }^{\circ} \mathrm{C}$ and $3.2 \mathrm{kPa}$ were selected as test conditions to study the deformation resistance of asphalt at high temperatures.

Figure 7 shows that the $J_{\mathrm{nr}}$ value of all asphalt decreases and the $R$ value increases with the aging time at $70{ }^{\circ} \mathrm{C}$ and $3.2 \mathrm{kPa}$, indicating that the high-temperature deformation resistance of asphalt gradually increases with increasing aging time. The $J_{\mathrm{nr}}$ value of JL70\# is larger than that of TPC70\# when they are not aged, and there is little difference between them. With the increase of aging time, the $J_{\mathrm{nr}}$ value of TPC70\# increases significantly after $120 \mathrm{~min}$, but the $R$-value of JL70\# has no obvious change, which indicates that TPC70\# has better anti-aging ability than JL70\#. The $R$-value of SBS-1 decreases rapidly, whereas the $R$-value of SBS-2 has a fluctuation region and does not change much, which indicates that SBS-2 has better resistance to deformation at high temperatures than SBS-1. Within the aging time of $0 \sim 360 \mathrm{~min}$, the $J_{\mathrm{nr}}$ of modified asphalt is always much smaller than that of base asphalt, and its recovery rate $R$ is much larger than that of base asphalt. That is to say, after aging, its resistance to deformation at high temperatures gradually increases, and the resistance to permanent deformation of modified asphalt is still significantly better than that of base asphalt, which is consistent 
with the analysis results of unaged asphalt samples. The order of resistance to deformation at a high temperature for the four types of asphalt is SBS-2 > SBS-1 > TPC 70\# > JL70\#.
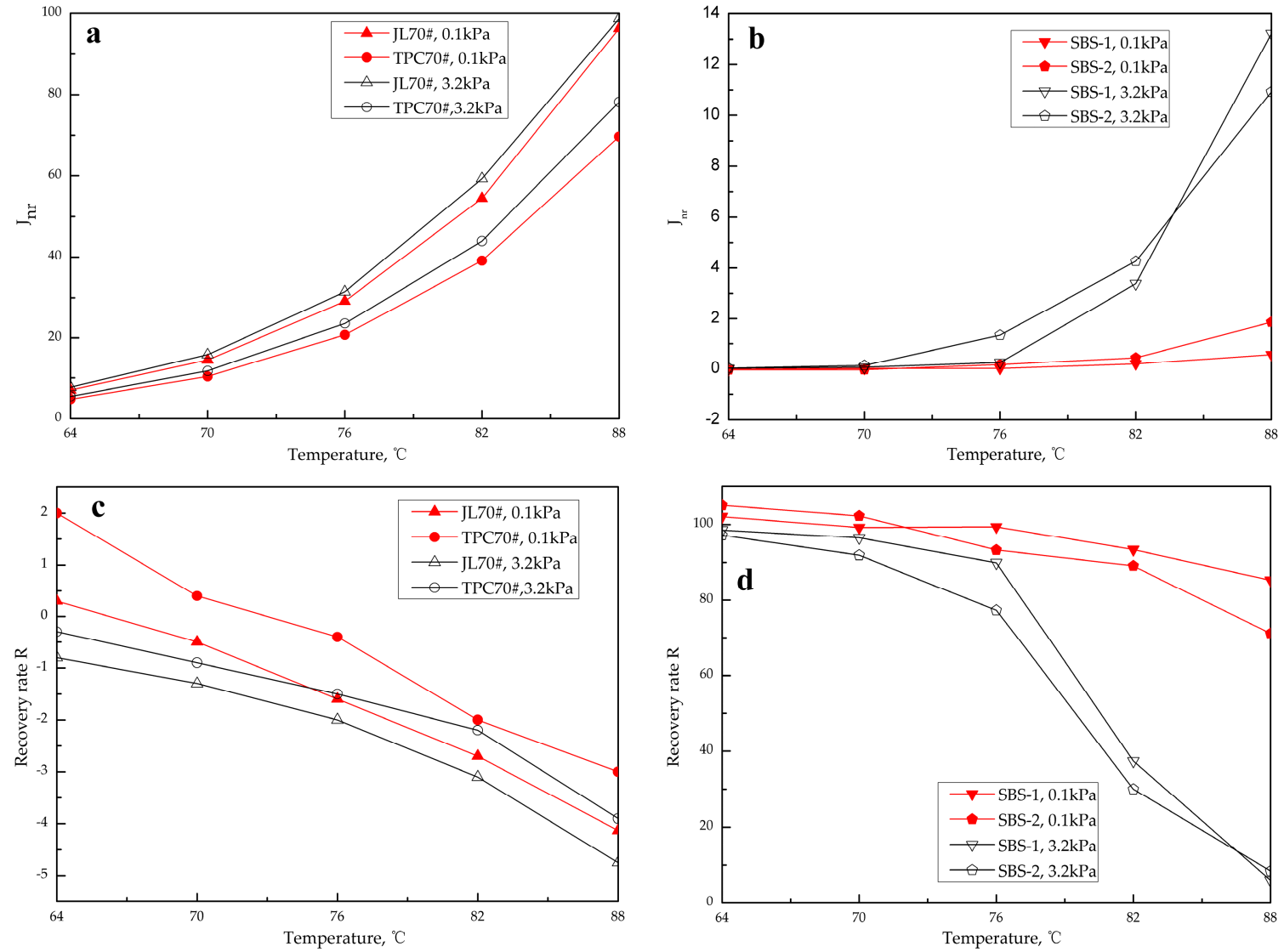

Figure 6. The $J_{\mathrm{nr}}$ of matrix asphalts (a) and modified asphalts (b), and the $R$ of matrix asphalts (c) modified asphalts (d) under different temperatures and stress conditions.
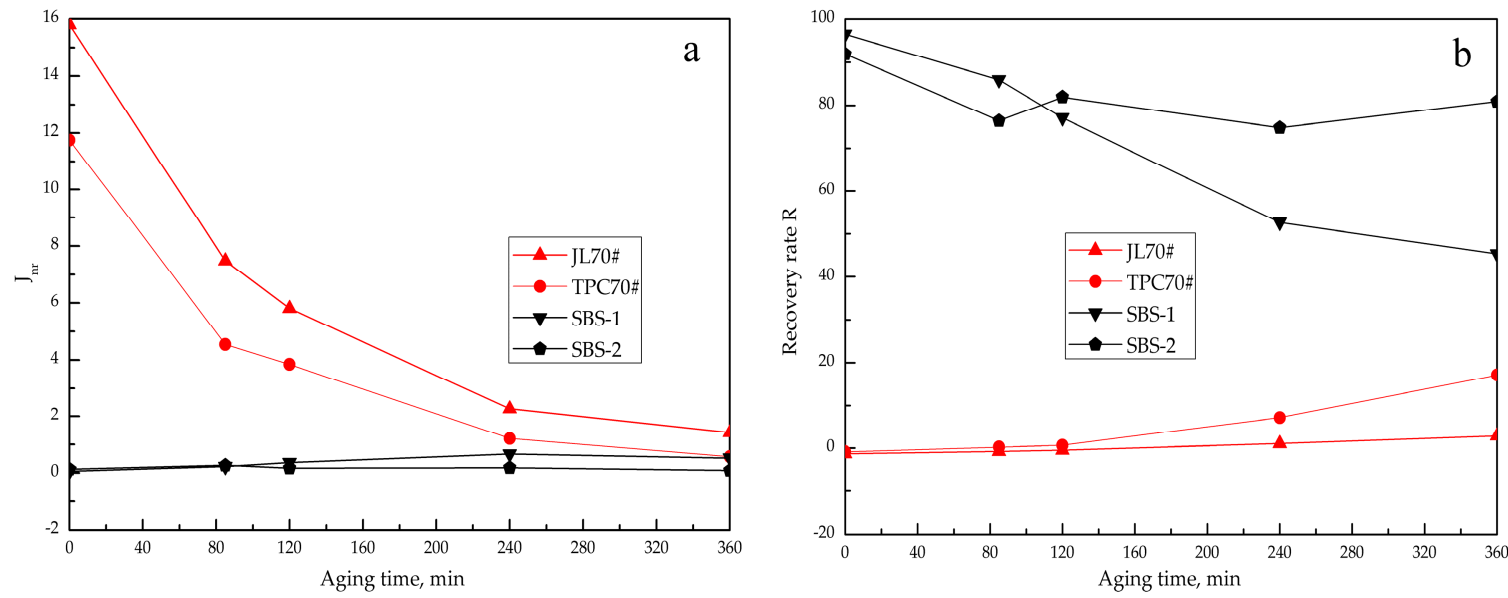

Figure 7. The $J_{\mathrm{nr}}(\mathbf{a})$ and $R(\mathbf{b})$ of asphalts $\left(70^{\circ} \mathrm{C}, 3.2 \mathrm{kPa}\right)$.

\subsection{Correlation Analysis between Functional Group Index and MSCR Test Results}

The quantitative analysis standard proposed in Section 3.1.2 was used to calculate the five characteristic functional group indices of 20 kinds of aged asphalt spectra. Based on the Levenberg-Marquardt method [31] and general global optimization algorithm, IBM SPSS Statistics 23 [32] was used to carry out multivariate statistical regression analysis on the asphalt recovery rate $R$ 
of the MSCR test under different temperatures and controlled stress and the functional group change index of infrared spectrum test. $R$ can be predicted by the following mathematical model:

$$
R=\alpha_{1}+\beta_{1} I_{\mathrm{C}=\mathrm{O}}+\beta_{2} I_{\mathrm{S}=\mathrm{O}}+\beta_{3} I_{\mathrm{B}, \alpha}+\beta_{4} I_{\mathrm{B}}+\beta_{5} I_{\mathrm{Ar}}
$$

where $\alpha_{1}, \beta_{1}, \beta_{2}, \beta_{3}, \beta_{4}, \beta_{5}$ are regression constants-the regression comparison results are shown in Figure 8 ( $R$ is the measured value and $R^{\prime}$ is the predicted value). The regression results were tested by the F-Statistic (see Table S5 in supplementary file Tables.pdf), in which $R^{2}$ is the correlation coefficient, MS is the mean square deviation, and Sig is the significance index.

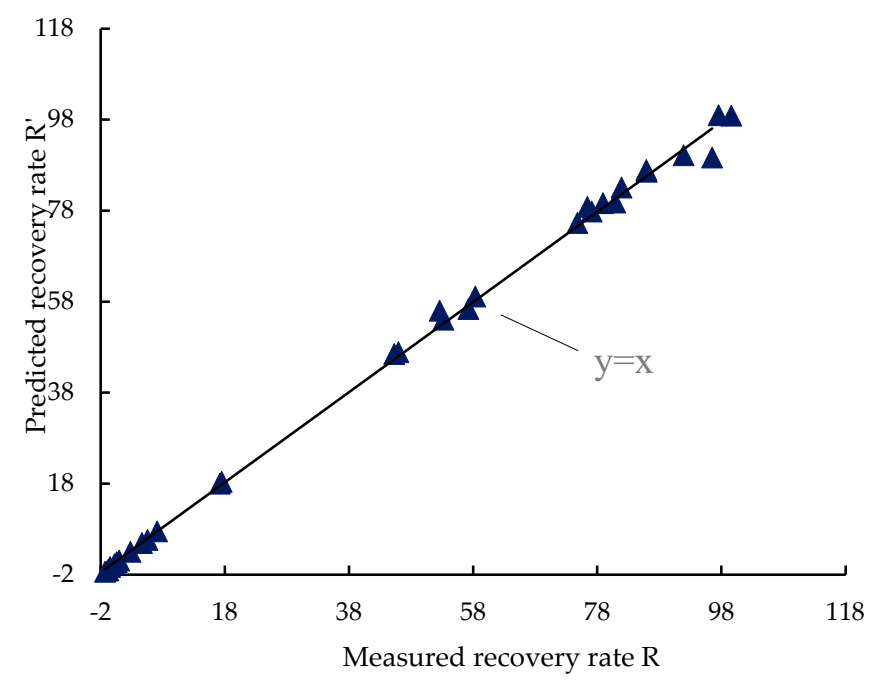

Figure 8. The prediction result of the aging asphalt recovery rate.

Table S5 shows that the recovery rate $R$ prediction model at different temperatures has a high accuracy correlation coefficient, and the significance is less than 0.05 . It passes the $F$ test, which shows that the asphalt's resistance to deformation at high temperature shows a multivariate linear relationship with the change of chemical functional group content. In Figure 8, the measured recovery rate $R$ is taken as the $x$-axis, and the recovery rate $R^{\prime}$ predicted by the five characteristic functional group indices is taken as the $y$-axis. The results show that the data points are located near the line $y=x$, indicating that the prediction model of the recovery rate $R$ of aged asphalt has good reliability.

\subsection{Establishment and Verification of Prediction Model of Asphalt Aging Time}

\subsubsection{Model Establishment}

Compared with the PLS and LDA methods, the PCA method is used to reduce data dimensionality and can solve the problem of spectral matrix collinearity and the limitation of the number of variables to a certain extent [22]. It is the most widely used method for data dimension reduction. The combination of infrared spectroscopy and PCA can extract more effective information in infrared spectroscopy measurements, thereby establishing a stable and highly reliable prediction model.

According to results of the quantitative analysis of the mid-infrared spectroscopy in Section 3.1.2, the PCA of the five main characteristic absorption peak functional group index values of 20 asphalt samples was performed using IBM SPSS Statistics 23.0 software (IBM Corp. Released 2015. IBM SPSS Statistics for Windows, Version 23.0. Armonk, NY: IBM Corp). The results are shown in Tables S6-S8 (see supplementary file Tables.pdf). Table S6 (see supplementary file Tables.pdf) shows that the correlation between the original variables is relatively high. The correlation between the aliphatic functional group index and the asymmetric aliphatic functional group index is as high as 0.946 , and the correlation between the carbonyl index and the sulfoxide functional group index also reaches 0.842 ; therefore, it is necessary to establish a PCA model. 
Table S7 (see supplementary file Tables.pdf) shows that the eigenvalues of the two components are greater than 1, and the cumulative contribution is as high as $81.43 \%$; therefore, selecting the first two components as the main components can represent most of the information of the original five data indicators. Table S8 (see supplementary file Tables.pdf) shows that $I_{\mathrm{B}, \mathrm{a}}, I_{\mathrm{B}}$, and $I_{\mathrm{Ar}}$ have a large load on PCA1 by analyzing the rotation component matrix, i.e., PCA1 characterizes the changes of saturated and aromatic components. According to the four-component analysis method, PCA1 can be defined as a "component change factor". $I_{\mathrm{C}=\mathrm{O}}$ and $I_{\mathrm{S}=\mathrm{O}}$ have a greater load on PAC2. $I_{\mathrm{C}=\mathrm{O}}$ and $I_{\mathrm{S}=\mathrm{O}}$ are the functional indices of carbonyl and sulfoxide groups, respectively, so PCA2 reflects the changes of functional indices of carbonyl and sulfoxide groups, and the changes of carbonyl and sulfoxide groups are caused by the absorption of oxygen and oxidation of asphalt. Therefore, PCA2 can be defined as the "oxidation factor".

The results of the principal component analysis of 20 asphalt samples with different aging degrees show that when the asphalt ages, PCA1 and PCA2 can represent the majority of the information on functional group changes in the infrared spectra. That is to say, they can both represent the change of the characteristic absorption peak of the attenuated total reflection infrared spectrum of aging asphalt, which can reflect the aging degree of asphalt to a certain extent.

$$
\begin{gathered}
\text { PCA1 }=0.15 Z X 1+0.21 Z X 2+0.38 Z X 3+0.34 Z X 4+0.28 Z X 5 \\
\text { PCA2 }=0.53 Z X 1+0.49 Z X 2-0.18 Z X 3-0.13 Z X 4-0.23 Z X 5 \\
F=\frac{49.35}{81.43} \times \text { PCA } 1+\frac{32.08}{81.43} \times \text { PCA2 }
\end{gathered}
$$

where ZX1 ZX5 are the standardized data of $I_{\mathrm{C}=\mathrm{O}}, I_{\mathrm{S}=\mathrm{O}}, I_{\mathrm{B}, \mathrm{a}}, I_{\mathrm{B}}$, and $I_{\mathrm{Ar}}$ functional group indices of each asphalt sample.

According to Equation (9), the comprehensive index $F$ value of each asphalt sample was calculated and analyzed. It was found that the comprehensive index $F$ value increases with the increasing aging; therefore, the aging of asphalt can be characterized by the comprehensive index $F$. The prediction model of $F$ and asphalt aging time was established as follows:

$$
\begin{gathered}
F_{\text {matrix asphalts }}=0.0048 \mathrm{t}-0.2518 \\
F_{\text {modified asphalts }}=0.0028 \mathrm{t}-0.9293
\end{gathered}
$$

Figure 9 shows that the $F$ value of asphalt has a linear relationship with the aging time of $0 \sim 360 \mathrm{~min}$, and the correlation degree is high. The $R^{2}$ (correlation coefficient) of the two matrix asphalts is 0.9508 , although those of the modified asphalts are slightly lower, mainly because the addition of modifiers makes the aging process more complicated and has more positive influence factors than the matrix asphalt.

\subsubsection{Model Validation}

The existing SK90\# matrix asphalt and its SBS modified asphalt with known aging time were predicted by the above prediction model, and the steps are as follows:

The infrared spectra of six groups of asphalt samples were collected by attenuated total reflection infrared spectrometer, and the measured infrared spectral data were preprocessed according to Section 3.1. Selecting A1 as the basis reference, the spectral data after pretreatment were quantitatively analyzed, and the functional index of five characteristic functional groups was obtained and standardized. The comprehensive index $F$ was calculated according to Equations (7)-(9). By substituting the comprehensive index $F$ value into the prediction model, the predicted aging time histories of six asphalt samples were obtained. Figure 10 shows that all data points are near $y=x$, and the variability of data at 120,240 , and $360 \mathrm{~min}$ is $6.6 \%, 4.0 \%$, and $6.2 \%$, respectively, which shows that the prediction model is reliable. 


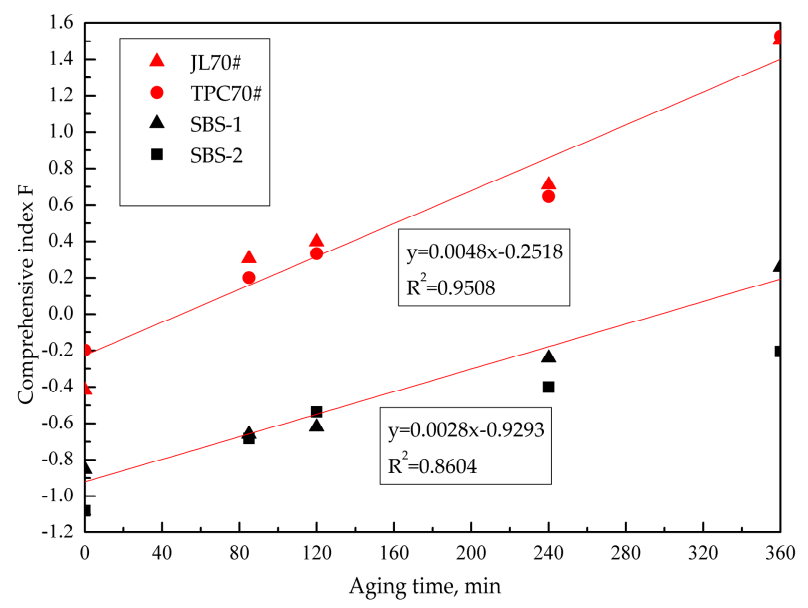

Figure 9. The linear relationship between $F$ and aging time.

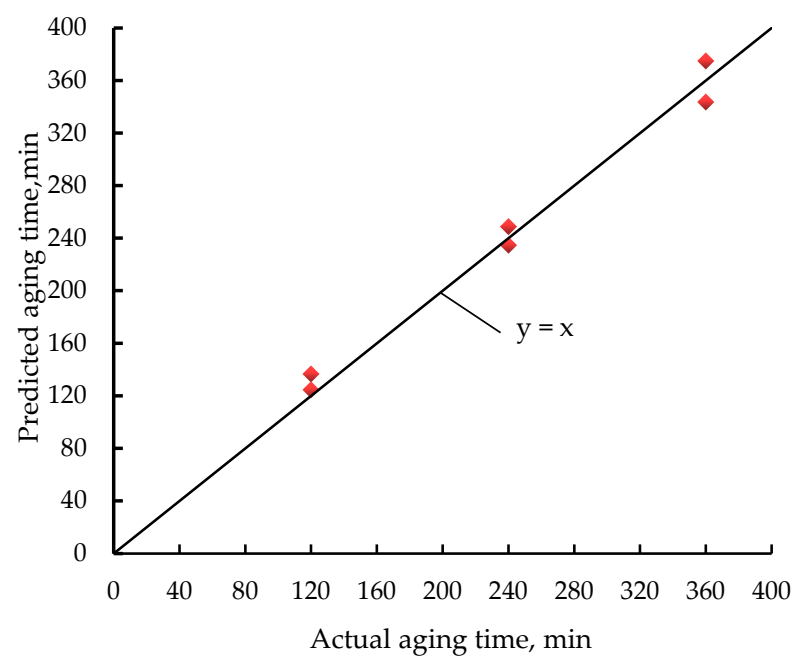

Figure 10. The validation of the asphalt aging time prediction model.

\subsection{Fatigue Life Analysis of Aging Asphalt Based on Infrared Spectroscopy}

\subsubsection{Establishment of Relationship Model between Fatigue Life and Aging Time of Asphalt}

The aging of asphalt is the main cause of its fatigue performance decay, and fatigue life is usually used to evaluate the fatigue cracking resistance of asphalt [33]. Fatigue life refers to the loading period required for fatigue failure of asphalt in time-scanning tests. At present, there are many methods available to define the loading period, and the fatigue life under different definition methods is different; therefore, choosing the appropriate definition method of fatigue life is the key to accurately analyze the fatigue cracking resistance of aging asphalt. A large number of studies show that the fatigue life determined by the the rate of dissipated energy change can accurately characterize the anti-fatigue performance of asphalt [34]. The rate of dissipated energy change (RDEC) is a fatigue life evaluation index based on the law of energy change. It evaluates the development process of fatigue damage according to the speed of dissipated energy change of asphalt in the process of loading. The RDEC curve is roughly divided into three stages: it drops sharply from the initial stage to the gentle fluctuation stage, and finally reaches the stage of rapid increase. The loading period corresponding to the sudden increase inflection point of the dissipation energy change rate curve is the asphalt fatigue life, which is recorded as $N_{\mathrm{RDEC}}$, and the calculation formula of the rate of dissipated energy change is as follows:

$$
\operatorname{RDEC}_{a}=\frac{\left|\omega_{a}-\omega_{b}\right|}{\omega_{a}(b-a)}
$$




$$
\omega_{a}=\pi \varepsilon \sigma \sin \delta=\pi \varepsilon^{2} G^{*} \sin \delta
$$

where $R D E C_{a}$ is the average rate of dissipated energy change of the $a$-th loading cycle relative to the $b$-th loading cycle; $\omega_{a}$ and $\omega_{b}$ are the dissipated energy corresponding to the $a$-th and $b$-th loading cycles, respectively, $G^{*}$ is the complex shear modulus, $\varepsilon, \delta$ are strain and stress, respectively.

To obtain the inflection point of RDEC curve, the RDEC needs to be further processed, and the calculation formula is as follows:

$$
\operatorname{RDEC}^{\prime \prime}=\frac{\mathrm{RDEC}_{b}-\mathrm{RDEC}_{a}}{\operatorname{RDEC}_{a}(b-a)}
$$

With $N_{\text {RDEC }}$ as the evaluation index, the fatigue life of four types of asphalt under the same aging time was compared (see Figure 11). It can be seen that the fatigue life of four kinds of asphalt is ranked as SBS- $1>$ JL7O\# > TPC70\# > SBS- 2 when the aging time is 0 and $85 \mathrm{~min}$. The modified asphalt SBS- 1 has the largest anti-fatigue performance, mainly because the asphalt has been aged during transportation and the hardness of the asphalt has increased, so its anti-fatigue performance is greater than that of matrix asphalt; however, after aging for $120 \mathrm{~min}$, the order is TPC70\# > SBS-1 > JL70\# > SBS-2, and after aging for 240 and $360 \mathrm{~min}$, the order is TPC70\# > JL70\# > SBS-2 > SBS-1. The changing orders show that with the increase of aging time, the fatigue resistance of matrix asphalt is greater than that of modified asphalt, among which the fatigue resistance of TPC70\# asphalt increases rapidly, exceeding JL70\#, while SBS-1 increases slowly, and finally is lower than that of SBS-2. The anti-fatigue performance of asphalt reflects the anti-aging ability of asphalt to a certain extent; therefore, the anti-aging ability of four types of asphalt is ranked as SBS-1 > SBS-2 > JL70\# > TPC70\#.

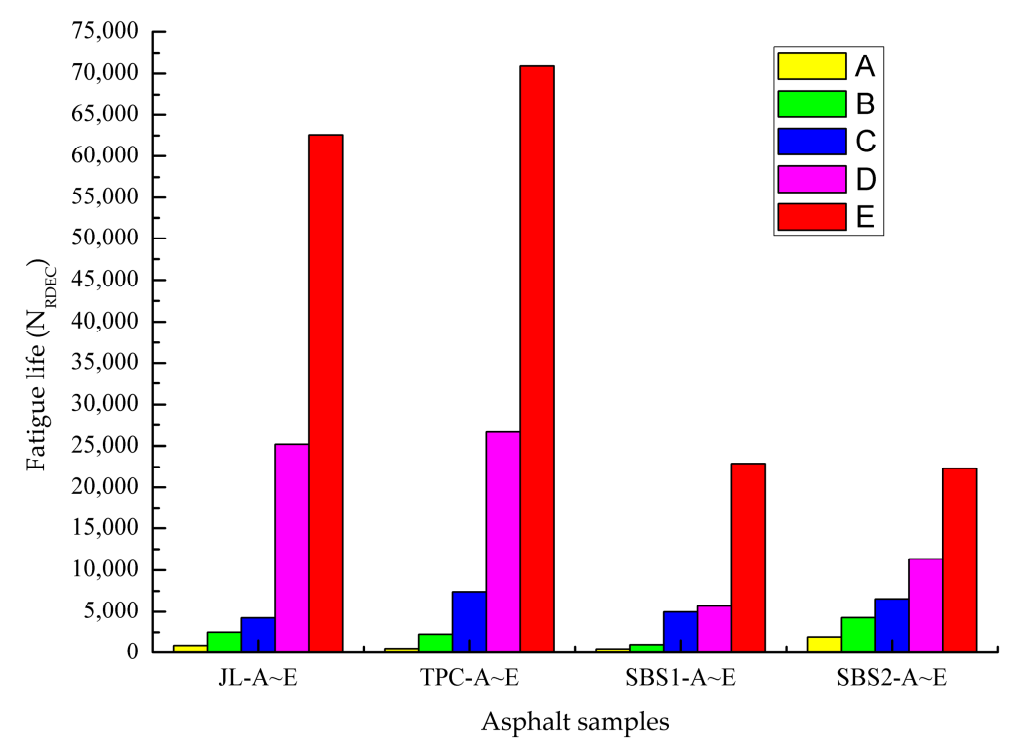

Figure 11. The fatigue life $\left(N_{\mathrm{RDEC}}\right)$ of all asphalt samples.

According to the analysis of the fatigue life change law of asphalt with different aging time, Figure 11 shows that the fatigue life of four types of asphalt increases with an aging time of 0-360 min, i.e., the fatigue resistance of asphalt increases with the aging time. Figure 12 shows the relationship between fatigue life and aging time. It reflects that the fatigue life of aged asphalt is exponentially correlated with aging time, and the correlation degree $R^{2}$ reached 0.86 , which proves that the longer the aging time of asphalt, the greater the fatigue life of the asphalt. The mathematical model is as follows:

$$
L n N_{R D E C}=\alpha T+\beta
$$

where $\alpha$ and $\beta$ are coefficients determined by experiments; $N_{\text {RDEC }}$ is the fatigue life determined by $\mathrm{RDEC} ; T$ is the aging time. 


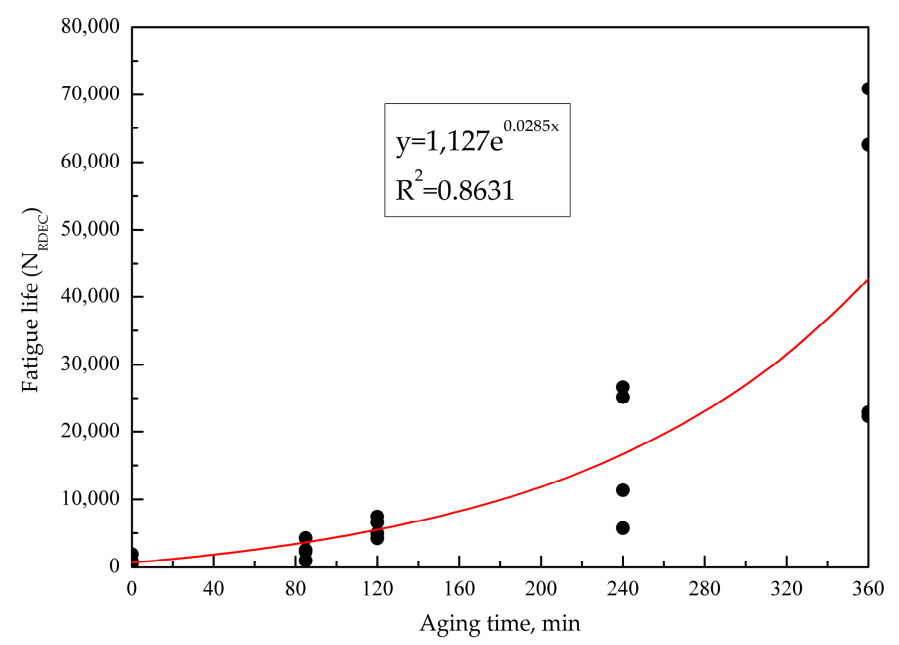

Figure 12. The relationship between fatigue life $N_{\text {RDEC }}$ and aging time.

\subsubsection{Correlation Analysis between Comprehensive Index F and Fatigue Life}

According to Section 3.5.1, the aging time of asphalt is linearly related to the logarithm of its fatigue life. There is a good linear correlation between asphalt aging time and asphalt comprehensive index F (see Section 3.4.1); therefore, there must be a certain relationship between the fatigue life of aging asphalt and its infrared spectrum PCA result. The fitting result between $N_{\text {RDEC }}$ and $F$ is shown in Figure 13, which shows that there is a positive correlation between them, i.e., with the increase of comprehensive index $F$, the $N_{\text {RDEC }}$ value also increases, and the correlation degree $R^{2}$ is 0.85 ; therefore, the fatigue life $N_{\mathrm{RDEC}}$ of asphalt can be predicted using the following mathematical model,

$$
N_{\mathrm{RDEC}}=\gamma_{1} F^{2}+\gamma_{2} F+\gamma_{3}
$$

where $\gamma_{1}, \gamma_{2}, \gamma_{3}$ are the constants determined by the tests.

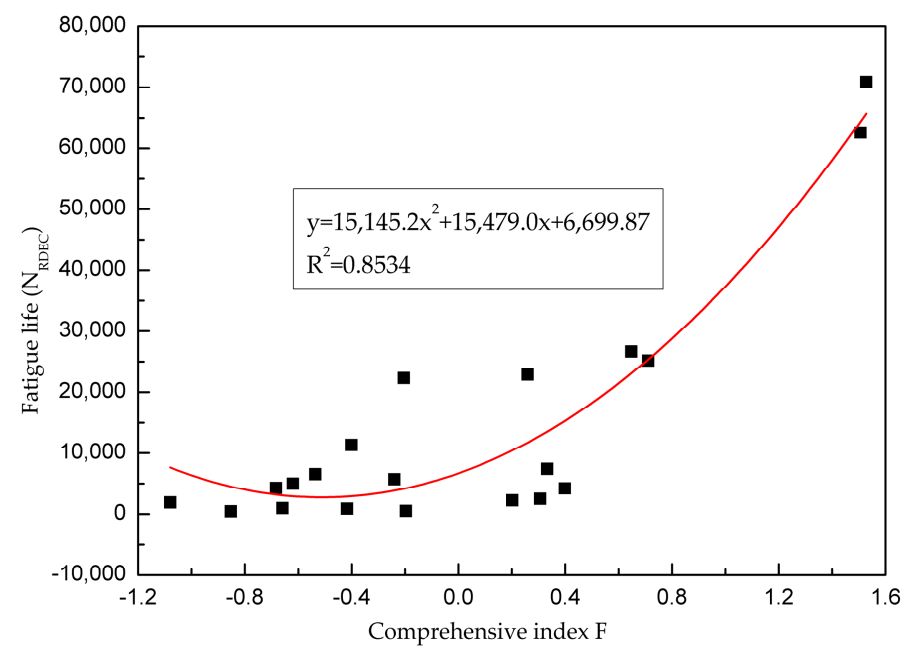

Figure 13. The fitting curve between $N_{\text {RDEC }}$ and $F$.

\section{Discussion}

FTIR, as one of the main methods used to characterize the aging properties of asphalt, has been applied by an increasing number of scholars to evaluate the structure and properties of asphalt [35]; however, the complexity of the asphalt structure and the instability and variability of performance of each component after being affected by the environmental damage have led to the diversity and disunity of asphalt research parameters. Researchers choose different evaluation methods, so the 
comparison of the absolute value of peak area in the infrared spectral data is not referential. The relative value changes are different with different parameters in the calculation process. The absolute value of the peak area for certain characteristic peaks of some aged asphalt are too small due to the large influence of parameters. To avoid the influence of experimental errors, human operation, and other factors, an increasing number of researchers choose a certain part of the peak value as a reference benchmark to calculate the change index of characteristic functional groups. Different benchmarks and different asphalt samples are selected to draw some similar conclusions about the change index of functional groups during asphalt aging, whereas others draw opposite conclusions; therefore, the research on asphalt aging characteristics is closely related to the artificially selected different asphalt samples and different benchmarks [24].

Figure 5 shows that the carbonyl index of the four types of asphalt shows an increasing trend under the selected four reference standards, whereas the sulfoxide group of Taipuke 70\# asphalt shows a negative growth within the aging time of 120-240 min under the benchmarks A2, A3, and A4, which does not conform to the aging mechanism of asphalt. Therefore, in this study, the peak value at A1 $\left(650 \sim 1400 \mathrm{~cm}^{-1}\right.$ ) was selected as the calculation benchmark to accurately and reasonably characterize the changes of functional groups during asphalt aging.

Qiu Longliang [36] used a coating film infrared testing method when studying the aging mechanism of SBS-modified asphalt, The methylene antisymmetric stretching vibration absorption peak at $2924 \mathrm{~cm}^{-1}$ was selected as a benchmark to calculate the peak area ratio, and the change of carbonyl index and sulfoxide indices were as follows: NEA (natural exposure aging) $>$ RTFOT $>$ unaged. When Zhao [37] used the same test method to analyze the aging characteristics of SBS- modified asphalt, he chose the methyl bending vibration absorption peak at $1460 \mathrm{~cm}^{-1}$ as the benchmark to calculate the peak area ratio and carry out infrared spectrum quantitative analysis, and the conclusion was consistent with Qiu.

Pang Ling [38] calculated the indices of functional groups at 1700, 1030, and $968 \mathrm{~cm}^{-1}$ based on the sum of peak areas between 2000 and $600 \mathrm{~cm}^{-1}$, and found that the indices of carbonyl and sulfoxide groups of AH-70 matrix asphalt changed in the following order: NEA > PAV (asphalt accelerated aging test) $>$ RTFOT $>$ unaged. Zhang [39] and others used infrared spectroscopy to study the changes of SK-90 asphalt before and after aging with SBS and SBS sulfur-modified asphalt and concluded that the change rule of the carbonyl index was the same as that of the above scholars, but the change rule of sulfoxide index was not obvious. Compared with the original one, the short-term thermal oxidation aging index increased, but most of them showed a decreasing trend when simulating PAV aging for 5 years. This demonstrates that the research on asphalt aging characteristics is a complex and important subject, which requires more scholars to develop new technologies and means to implement the research.

Finally, this work still contains some possibilities for further in-depth study. For example, in the process of establishing and verifying the prediction model, the types and quantities of selected asphalt samples need to be further enriched. This study realized the rapid prediction of asphalt aging time and fatigue life by infrared spectra, and further considered the integration of an infrared spectrum pretreatment process, quantitative analysis, principal component analysis, and prediction model, and designed an entire set of prediction systems. As long as the infrared spectrum is input, it is possible to get the prediction results directly by integrated software.

\section{Conclusions}

This work established a prediction model of asphalt aging time history and fatigue life, based on infrared spectroscopy technology and the PCA method. Firstly, the qualitative and quantitative analysis of the attenuated total reflection infrared spectrum after pretreatment was carried out. Then, the high-temperature deformation resistance of various aged asphalts was analyzed based on the infrared spectrum quantitative analysis and the relationship between the functional group index and the recovery rate $R$ was discussed. Taking the main component analysis results as original data, 
an asphalt aging time prediction model was established and verified. The fatigue life of various aged asphalt samples was analyzed by adopting $N_{\mathrm{RDEC}}$ as the fatigue life evaluation index. Finally, the correlation between the comprehensive index $F$ and asphalt fatigue life was explored in this work. The main research conclusions are as follows:

- After the asphalt is aged, its recovery rate $R$ increases, and the high-temperature resistance to deformation is enhanced. The high-temperature resistance to deformation of four types of asphalt are ranked as follows: SBS-2 > SBS-1 > TPC70\# > JL70\#. The recovery rate $R$ and the functional group index showed a multivariate linear correlation and a high degree of correlation. Figure 8 shows that the data points of the recovery rate $R$ predicted by the five characteristic functional group indices are all located near the straight line of $y=x$, indicating the aging based on the functional group index. The prediction model of the asphalt recovery rate $R$ has good reliability and can achieve rapid prediction of aging asphalt high-temperature resistance to deformation to a certain extent.

- The PCA method was used to determine two principal component factors, namely the oxidation factor and component change factor, both of which can be used to characterize the aging degree of asphalt. The asphalt aging time prediction model based on the principal component comprehensive evaluation index $F$ has a certain degree of reliability, indicating that the asphalt infrared spectroscopy can quickly predict its aging time.

- Taking $N_{\mathrm{RDEC}}$ as the fatigue life evaluation index, the fatigue life rankings of the four types of asphalt are: SBS-1 $>$ SBS-2 $>$ JL70\# $>$ TPC70\#, and $N_{\text {RDEC }}$ is exponentially correlated with the aging time-the correlation (squared) reaches 0.86 . The fatigue life of aging asphalt is positively correlated with the comprehensive index $F$ of asphalt, and the correlation degree $R^{2}$ is 0.85 , i.e., the comprehensive index $F$ of asphalt increases when its fatigue life also increases; therefore, it is feasible to use infrared spectroscopy to predict the aging time and fatigue life of asphalt, and it can provide a rapid and non-destructive prediction method for the practical application of asphalt.

Supplementary Materials: The following are available online at http://www.mdpi.com/2079-6412/10/10/959/s1, Table S1: Basic physical performance indices of asphalt for the four samples, Table S2: Aging asphalt samples, Table S3: The values of spectral peak area under each benchmark, Table S4: Functional group index of aged asphalt, Table S5: Fitting results of the recovery rate R model, Table S6: Correlation matrix of five characteristic functional group indices, Table S7: Total variance explanation, Table S8: Rotating component matrix.

Author Contributions: Conceptualization, X.Z. and Y.Y. (Yuan Yan); Methodology, Y.Y. (Yuan Yan), M.R., and Y.Y. (Yanmei Yang); Software, Y.Y. (Yuan Yan); Validation, X.Z., M.R. and Y.Y. (Yanmei Yang); Formal Analysis, M.G., and Y.Y. (Yuan Yan); Investigation, Y.Y. (Yanmei Yang); Resources, M.G. And Y.Y. (Yuan Yan); Data Curation, Y.Y. (Yanmei Yang); Writing-Original Draft Preparation, Y.Y. (Yuan Yan), and M.R.; Writing-Review \& Editing, Y.Y. (Yuan Yan), M.R., and Y.Y. (Yanmei Yang); Visualization, L.Z., and M.G.; Supervision, X.Z., and M.G.; Project Administration, X.Z., and L.Z.; Funding Acquisition, X.Z., and L.Z. All authors have read and agreed to the published version of the manuscript.

Funding: This research was funded by the National Natural Science Foundation of China (NSFC; 51827812, 51778509, 51578430), the Hubei Provincial Natural Science Foundation of China (2018CFB293), and the overseas study program for young teachers of Hubei Province (201659194).

Acknowledgments: The first author would like to give her most sincere thanks to Ulf Standberg, who is a respected senior researcher of the Swedish National Road and Transport Research Institute (VTI). He has read the entire manuscript and given useful language and technical comments.

Conflicts of Interest: The authors declare no conflict of interest.

\section{References}

1. Hou, X.; Lv, S.; Chen, Z.; Xiao, F. Applications of fourier transform infrared spectroscopy technologies on asphalt materials. Measurement 2018, 121, 304-316. [CrossRef]

2. Kleizienè, R.; Panasenkienè, M.; Vaitkus, A. Effect of Aging on Chemical Composition and Rheological Properties of Neat and Modified Bitumen. Materials 2019, 12, 4066. [CrossRef] [PubMed] 
3. Chu, X.L.; Xu, Y.P.; Lu, W.Z. Research and application progress of chemometrics for near infrared spectroscopy. Anal. Chem. 2008, 5, 702-709.

4. Zhang, Z.Y.; Shen, J.N.; Shi, P.C.; Zhu, H. Micro-mechanism of asphalt aging based on nano-mechanics and functional groups. Highw. Traffic Sci. Tech. 2017, 34, 19-27.

5. Li, P.; Nian, T.F.; Wei, D.B.; Lin, M. FTIR quantitative analysis method and new exploration of rheological parameters of aged asphalt. J. Huazhong Univ. Sci. Tech. Nat. Sci. Ed. 2018, 46, 34-39.

6. Wang, Y.B.; Luo, A.L.; Lu, W.Z.; Yuan, H.F. Rapid determination of wax content in asphalt by near infrared analysis. Acta Petrolei. Sinica Pet. Eng. 2001, 3, 68-72.

7. Tang, J.Q. Research on Rapid Identification and Analysis of Asphalt Materials. Master's Thesis, Beijing University of Chemical Technology, Beijing, China, 2015.

8. Wang, Y.J.; Chen, Q.T.; Zhao, W. Study on Rapid Detection of Asphalt Properties by Attenuated Total Reflection Infrared Spectroscopy. Highw. Traffic Tech. Appl. Tech. Ed. 2016, 12, 74-77.

9. Nivitha, M.R.; Prasad, E.; Krishnan, J.M. Aging in modified bitumen using FTIR spectroscopy. Int. J. Pavement Eng. 2016, 17, 565-577. [CrossRef]

10. Ye, Y.F.; Zhen, Y.; Zhang, X.R.; Wu, H.N. Study on rapid detection method of asphalt penetration. Pet. Ref. Chem. Ind. 2014, 6, 13-16.

11. Weigel, S.; Stephan, D. The prediction of bitumen properties based on FTIR and multivariate analysis methods. Fuel 2017, 208, 655-661. [CrossRef]

12. Hao, P.L. Evaluation and Analysis of Aging Detection of Asphalt Mixture for Road Use. North. Trans. 2015, 7,71-73.

13. Weigel, S.; Stephan, D. Bitumen Characterization with Fourier Transform Infrared Spectroscopy and Multivariate Evaluation: Prediction of Various Physical and Chemical Parameters. Energy Fuels 2018, 32, 10437-10442. [CrossRef]

14. Zeng, M.; Pan, H.; Zhao, Y.; Tian, W. Evaluation of asphalt binder containing castor oil-based bioasphalt using conventional tests. Constr. Build. Mater. 2016, 126, 537-543. [CrossRef]

15. Siddiqui, M.; Ali, M. Studies on the aging behavior of the Arabian asphalts. Fuel 1999, 78, $1005-1015$. [CrossRef]

16. Lin, L.; Li, X.L.; Zheng, G.Y.; Zhang, L.Q. New discussion on the relationship between aging performance of petroleum asphalt and its chemical composition. Mater. Guide 2013, 27, 123-126.

17. Ma, Z. Performance evaluation of sulfur modified asphalt based on DSR test. Traffic Sci. Eng. 2017, 33, 23-26.

18. Huo, Y.; Zhu, J.; Li, J.; Li, G.; Li, H. An active La/TiO2 photocatalyst prepared by ultrasonication-assisted sol-gel method followed by treatment under supercritical conditions. J. Mol. Catal. Chem. 2007, 278, 237-243. [CrossRef]

19. JTGE20-2011. Testing Regulations for Asphalt and Asphalt Mixtures of Highway Engineering; Highway Research Institute of the Ministry of Communications: Beijing, China, 2011.

20. ASTM D7405-20. Standard Test Method for Multiple Stress Creep and Recovery (MSCR) of Asphalt Binder Using a Dynamic Shear Rheometer; ASTM International: West Conshohocken, PA, USA, 2020. [CrossRef]

21. Bahia, H.U.; Hanson, D.I.; Zeng, M.; Zhai, H.; Khatri, M.A.; Anderson, R.M. Characterization of Modified Asphalt Binders in Superpave Mix Design; NCHRP Report 459; Transportation Research Board, National Research Council: Washington, DC, USA, 2001.

22. Lamontagne, J.; Dumas, P.; Mouillet, V.; Kister, J. Comparison by Fourier transform infrared (FTIR) spectroscopy of different ageing techniques: Application to road bitumens. Fuel 2001, 80, 483-488. [CrossRef]

23. Zhang, B.L. Study on Asphalt Structure Characterization Based on Infrared Spectroscopy. Master's Thesis, Wuhan University of Technology, Wuhan, China, 2014.

24. Yao, H.; Dai, Q.; You, Z. Fourier Transform Infrared Spectroscopy characterization of aging-related properties of original and nano-modified asphalt binders. Constr. Build. Mater. 2015, 101, 1078-1087. [CrossRef]

25. Zhang, D.; Zhang, H.; Shi, C. Investigation of aging performance of SBS modified asphalt with various aging methods. Constr. Build. Mater. 2017, 145, 445-451. [CrossRef]

26. Yut, I.; Zofka, A. Correlation between rheology and chemical composition of aged polymer-modified asphalts. Constr. Build. Mater. 2014, 62, 109-117. [CrossRef]

27. Gobrecht, A.; Bendoula, R.; Roger, J.M.; Bellon-Maurel, V. Combining linear polarization spectroscopy and the representative layer theory to measure the beer-lambert law absorbance of highly scattering materials. Anal. Chim. Acta 2015, 853, 486-494. [CrossRef] [PubMed] 
28. Chen, Y.D.; Tu, J.; Zhang, B.; Ding, H. Study on the influencing factors of SBS modified asphalt by infrared spectroscopy. Pet. Asphalt. 2014, 28, 67-72.

29. Liu, B.; Shen, J.N.; Shi, P.C. Nano-scale microscopic characteristics and functional group properties of aged asphalt. Highw. Traffic Sci. Tech. 2016, 33, 6-13.

30. Hofko, B.; Porot, L.; Cannone, A.F.; Poulikakos, L.; Huber, L.; Lu, X.; Mollenhauer, K.; Grothe, H. FTIR spectral analysis of bituminous binders: Reproducibility and impact of ageing temperature. Mater. Struct. 2018, 51, 45. [CrossRef]

31. Song, Z.F.; Wang, J.; Li, J. Levenberg-Marquardt Algorithm for Orthogonal Fitting of Transition Curve. J. Southwest Jiaotong Univ. 2020, 55, 144-149.

32. George, D.; Mallery, P. IBM SPSS Statistics 23 Step by Step: A Simple Guide and Reference; Routledge: New York, NY, USA, 2016.

33. Lu, W.Z. Modern Near Infrared Spectroscopy, 2nd ed.; Sinopec Press: Beijing, China, 2007.

34. Luo, R.; Xu, Y.; Liu, H.Q.; Zhang, D.R.; Feng, G.L. Rheological mechanical properties of DCLR modified asphalt. China J. Highw. Trans. 2018, 31, 165-171.

35. Zhou, X.L.; Yang, Y.M.; Guan, J.X.; Yan, Y. Prediction of asphalt aging time history based on infrared spectrum. Sino-Foreign Highw. 2020, 40, 218-223.

36. Qiu, L.L. Study on Aging and Regeneration Mechanism of SBS Modified Asphalt. Master's Thesis, Dalian University of Technology, Dalian, China, 2012.

37. Zhao, Y.L.; Gu, F.; Huang, X.M. Analysis of aging characteristics of SBS modified asphalt based on FTIR. J. Build. Mater. 2011, 14, 620-623.

38. Pang, L. Study on UV Aging Characteristics of Asphalt. Ph.D. Thesis, Wuhan University of Technology, Wuhan, China, 2008.

39. Zhang, F.; Yu, G.Y.; Han, J. The Effects of thermal oxidative aging on dynamic viscosity, TG/DTG, DTA and FTIR of SBS-and SBS sulfur-modified asphalts. Constr. Build. Mater. 2011, 25, 129-137. [CrossRef]

Publisher's Note: MDPI stays neutral with regard to jurisdictional claims in published maps and institutional affiliations.

(C) 2020 by the authors. Licensee MDPI, Basel, Switzerland. This article is an open access article distributed under the terms and conditions of the Creative Commons Attribution (CC BY) license (http://creativecommons.org/licenses/by/4.0/). 